\title{
Offline Signature Verification using Intelligent Algorithm
}

\author{
Kamlesh Kumari, Sanjeev Rana \\ Deptt. of CSE, M. M. University, Mullana, Ambala \\ *Corresponding author E-mails:savyakamlesh@gmail.com
}

\begin{abstract}
:-
Signature verification is important in banking, legal, financial transactions for security purpose. Offline signature verification is a complex task because active information i.e. temporal information is missing in static image. There is no standard feature extraction method for offline signature identification as in case of other behavior modalities e.g. in automatic speech recognition like LPCC (Linear Predictive Ceptral Coefficients).Our research presents an intelligent algorithm for feature extraction based on image difference of genuine signature image and questioned signature image. Six features i.e. average object area, entropy, standard deviation, mean, Euler no., and area are analyzed. Best results are reported using combination of Average Object Area, Mean, Euler No. and Area. CEDAR (Center of Excellence for Document Analysis) database is used for offline signature verification. The database consists of static signature samples taken from 55 users. The Proposed algorithm is quite efficient as it is less computationally. Experiments are performed with both models i.e. Writer-Independent (WI) system and Writer-Dependent.
\end{abstract}

Keywords: Center of Excellence for Document Analysis (CEDAR), K-nearest neighbor (kNN), Support Vector Machine (SVM), Writer-Independent (WI), Writer-Dependent (WD)

\section{Introduction}

Biometric system works by going through four phases. This includes image capturing phase, feature extraction phase, matching phase and decision phase. The sample is presented at sensor, which capture the unique information. This unique information is known as feature set. This is made into the form, which can be processed and known as template. This template is matched with stored database and decision is made to accept or reject the user. Physiological characteristics are related to the shape of the body.e.g Face, DNA. Behavioral modalities are related to human behavior that may change over time like signature, typing rhythm. Signature verification is important in banking, legal, financial transactions for security purpose .Proficient handwritten signature authentication system still plays a key role in data protection. Forensic Document Expert evaluates features in signature based on the abrupt change in the direction of strokes, pen lift, pen pause, letter forms etc. Letter forms are the important features for examination of signature. For legal and social acceptance, handwritten signature is used for personal authentication. Offline signature verification has been an intense research field. Forensic Science Institutes are in search of offline signature verification system that can be used in forensic analysis. Figure 1 shows Samples of three users.

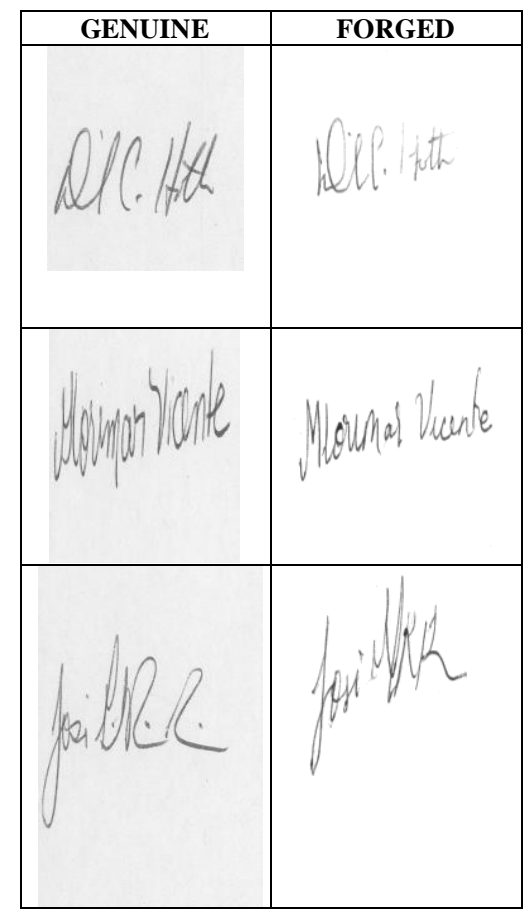

Fig.1: Samples of three users

\subsection{Advantages}

1. Hardware expenditure is less in offline signature verification system.

2. Offline signature verification will decrease the disruption to receive practices with respect to dealings where Personal verification has to be authenticated.

Due to geographic location, emotional state, age, posture, illness etc., there are some variations in the signatures of the same person. 
The paper is organized as follows: Section 1 describes the introduction. Section 2 presents the related work. Section 3 describes Architecture. Section 4 describes Experimentation. Section 5 presents the conclusion

\section{Related Work}

Each feature has its own significance and the contribution of discriminative feature affects the accuracy of offline signature verification system [1]. There is no standard feature extraction method for offline signature recognition as in case of automatic speech recognition [2]. Writer-Independent (WI) offline signature verification using feature extraction based on Average object area and entropy is proposed [3].Table 1 shows the features and classifier used by some of the researchers for offline signature verification.

\section{System Architecture:}

The figure 2 describes the architecture used for offline signature The input to system is the images of a genuine and a questioned signature. Output of the system determines whether questioned signature is Genuine Signature or Forged.

\subsection{Algorithm for feature selection:}

INPUT: Offline Signature image pair OUTPUT: Average Object Area, Mean, Euler No., Area Step 1: Resize the image pair.

Step 2: Calculate the absolute difference of image pair. Step 3: Calculate Average Object Area, Entropy, Standard Deviation, Mean, Euler No., and Area of the image obtained in Step-2

Step 4: Calculate the accuracy with combination of features obtained in Step 3 with proposed architecture.

Step 5:-Select the combination of features that gives more accuracy.

\subsection{Datasets Details:-}

Publically available database CEDAR [17] is used. The training data set contained offline signatures (24 original, 24 forged) of each of the 55writter. The database comprises of 1320 offline genuine images and 1320 offline forged signature images corresponding to 55 signers. The motivation was to test that system whether it works on input that it has never seen. Two forgeries differ from each other is not important to us. Table 2 shows the feature values extracted for Genuine Signature pair for one user. Table 3 shows Feature values extracted for Genuine Forged Signature pair for one user.

Table 1: Features used by researcher

\begin{tabular}{|l|l|l|l|}
\hline Feature Extraction & $\begin{array}{l}\text { Offline } \\
\text { Database }\end{array}$ & Classifier & Ref: \\
\hline $\begin{array}{l}\text { Histogram of } \\
\text { Templates (HOT) }\end{array}$ & CEDAR & SVM & 4 \\
\hline $\begin{array}{l}\text { Global and Local } \\
\text { Features }\end{array}$ & $\begin{array}{l}\text { Manipuri } \\
\text { signature }\end{array}$ & SVM & 5 \\
\hline $\begin{array}{l}\text { Histogram of curvature } \\
\text { (HOC) and histogram of } \\
\text { gradient (HOG) }\end{array}$ & UTSig & SVM & 6 \\
\hline Geometric and local & DB1 & Artificial & 7 \\
\hline
\end{tabular}

\begin{tabular}{|c|c|c|c|}
\hline binary pattern & & $\begin{array}{l}\text { Neural Network, } \\
\text { Support Vector } \\
\text { Machine }\end{array}$ & \\
\hline $\mathrm{HOG}$ & $\begin{array}{l}\text { 15-People } \\
\text { database }\end{array}$ & $\begin{array}{l}\text { GRNN(General } \\
\text { Regression Neural } \\
\text { Networks) }\end{array}$ & 8 \\
\hline $\begin{array}{l}\text { Pyramid } \\
\text { histogram of oriented } \\
\text { gradient (PHOG) }\end{array}$ & $\begin{array}{l}\text { FUM- } \\
\text { PHSDB }\end{array}$ & $\begin{array}{l}\text { modified K-nearest } \\
\text { neighbor }(\mathrm{MKNN})\end{array}$ & 9 \\
\hline KAZE & MCYT-75 & SVM & 10 \\
\hline $\begin{array}{l}\text { Invariant Directional } \\
\text { Feature }\end{array}$ & & $\begin{array}{l}\text { Longest Common } \\
\text { Subsequence } \\
\text { algorithm } \\
\text { (LCS) }\end{array}$ & 11 \\
\hline $\begin{array}{l}\text { KAZE } \\
\text { features in the BoVW } \\
\text { model }\end{array}$ & CEDAR & SVM & 12 \\
\hline ULBP & $\begin{array}{l}\text { BHSig260, } \\
\text { GPDS-100 }\end{array}$ & $\begin{array}{l}\text { Nearest Neighbour } \\
\text { (NN) Euclidian } \\
\text { distance }\end{array}$ & 13 \\
\hline $\begin{array}{l}\text { Entropy ,Eccentricity, } \\
\text { Standard Deviation, } \\
\text { Convex Area and } \\
\text { orientation }\end{array}$ & & $\begin{array}{l}\text { Artificial Neural } \\
\text { Networks ANNs): }\end{array}$ & 14 \\
\hline LDP,LBP, LDerivP & MCYT & $\begin{array}{l}\text { k-Nearest Neighbor } \\
\text { Classifier (KNNC), } \\
\text { Linear Discriminant } \\
\text { Classifier (LDC). }\end{array}$ & 15 \\
\hline Zernike moments & CEDAR & $\begin{array}{l}\text { Harmonic mean } \\
\text { dissimilarity } \\
\text { measure }\end{array}$ & 16 \\
\hline
\end{tabular}

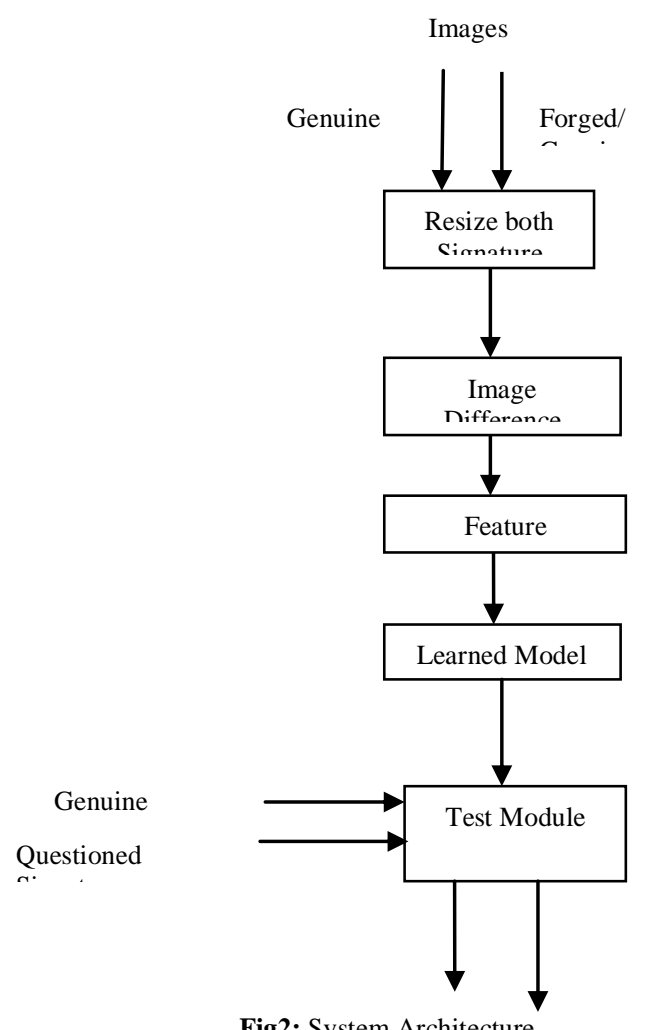

Fig2: System Architecture 
Table 2: Feature values extracted for Genuine Signature pair

\begin{tabular}{|r|r|r|r|r|r|}
\hline Entropy & $\begin{array}{c}\text { Average } \\
\text { Object area }\end{array}$ & $\begin{array}{c}\text { Standard } \\
\text { Deviation }\end{array}$ & Mean & \multicolumn{1}{c|}{$\begin{array}{c}\text { Euler } \\
\text { No. }\end{array}$} & \multicolumn{1}{c|}{ Area } \\
\hline 0.377511 & 16.04762 & 0.26037 & 0.073134 & -18 & 724 \\
\hline 0.380287 & 13.89796 & 0.261611 & 0.073893 & -14 & 730.75 \\
\hline 0.346965 & 10.89091 & 0.246532 & 0.064996 & -18 & 643.5 \\
\hline 0.392051 & 15.8 & 0.266841 & 0.077148 & -19 & 763.625 \\
\hline 0.373523 & 12.5283 & 0.258582 & 0.072049 & 5 & 708.625 \\
\hline 0.374322 & 14.17021 & 0.258941 & 0.072266 & -6 & 711.875 \\
\hline 0.361812 & 11.14035 & 0.253301 & 0.068902 & -9 & 682.25 \\
\hline 0.321402 & 8.693548 & 0.234671 & 0.058485 & -23 & 588.125 \\
\hline 0.333463 & 10.125 & 0.240301 & 0.061523 & -29 & 616.5 \\
\hline 0.396696 & 15.38298 & 0.268894 & 0.078451 & 0 & 765.625 \\
\hline 0.37192 & 15.34884 & 0.257863 & 0.071615 & -12 & 698.25 \\
\hline 0.352781 & 17.02778 & 0.249193 & 0.066515 & -26 & 660.625 \\
\hline 0.350295 & 9.790323 & 0.248057 & 0.065864 & -23 & 658.875 \\
\hline 0.317913 & 8.428571 & 0.233031 & 0.057617 & -21 & 577.875 \\
\hline 0.313081 & 11.81818 & 0.23075 & 0.056424 & -2 & 554.125 \\
\hline 0.408161 & 15.36735 & 0.273931 & 0.081706 & -29 & 808.25 \\
\hline 0.37192 & 9.041096 & 0.257863 & 0.071615 & -24 & 714.75 \\
\hline 0.369106 & 8.945205 & 0.256596 & 0.070855 & -13 & 703.875 \\
\hline 0.372722 & 12.49057 & 0.258223 & 0.071832 & -24 & 712.75 \\
\hline 0.3683 & 11.22414 & 0.256233 & 0.070638 & 0 & 695.5 \\
\hline 0.298804 & 7.625 & 0.223949 & 0.052951 & -4 & 525.5 \\
\hline 0.375121 & 9.970149 & 0.259299 & 0.072483 & -42 & 729.5 \\
\hline 0.335167 & 7.320513 & 0.241091 & 0.061957 & -14 & 620.75 \\
\hline & & & & & \\
\hline
\end{tabular}

Table 3: Feature values extracted for Genuine - Forged Signature pair

\begin{tabular}{|c|c|c|c|c|c|}
\hline Entropy & $\begin{array}{c}\text { Average } \\
\text { Object } \\
\text { area }\end{array}$ & $\begin{array}{c}\text { Standard } \\
\text { Deviation }\end{array}$ & Mean & $\begin{array}{c}\text { Euler } \\
\text { No. }\end{array}$ & Area \\
\hline 0.269661 & 9.042553 & 0.209746 & 0.046115 & -2 & 458 \\
\hline 0.3091 & 10.01961 & 0.228863 & 0.055447 & 3 & 547.75 \\
\hline 0.286028 & 8.846154 & 0.217777 & 0.049913 & 11 & 487.375 \\
\hline 0.282791 & 8.388889 & 0.2162 & 0.049154 & -6 & 489.25 \\
\hline 0.321837 & 10.8 & 0.234876 & 0.058594 & 9 & 575.875 \\
\hline 0.300608 & 7.6875 & 0.224813 & 0.053385 & 12 & 526.25 \\
\hline 0.280468 & 6.892308 & 0.215065 & 0.048611 & 18 & 477.875 \\
\hline 0.286489 & 6.779412 & 0.218002 & 0.050022 & 9 & 497.625 \\
\hline 0.279536 & 6.194444 & 0.214609 & 0.048394 & 31 & 474.125 \\
\hline 0.257198 & 7.388889 & 0.20353 & 0.043294 & 17 & 425.375 \\
\hline 0.351124 & 9.369231 & 0.248437 & 0.066081 & 29 & 640.125 \\
\hline 0.261061 & 8.847826 & 0.205467 & 0.044162 & 7 & 434.375 \\
\hline 0.268711 & 8.46 & 0.209276 & 0.045898 & 20 & 449.625 \\
\hline 0.295182 & 10.66667 & 0.222207 & 0.052083 & 17 & 508.625 \\
\hline 0.275324 & 11.5 & 0.212542 & 0.047418 & 20 & 462.125 \\
\hline 0.31966 & 9.067797 & 0.233853 & 0.058051 & 13 & 569 \\
\hline 0.282791 & 11.92105 & 0.2162 & 0.049154 & 12 & 479.5 \\
\hline 0.298353 & 10.3617 & 0.223732 & 0.052843 & 15 & 516.5 \\
\hline 0.250373 & 7.403846 & 0.200086 & 0.041775 & 13 & 410.75 \\
\hline 0.297901 & 9 & 0.223515 & 0.052734 & 19 & 513.5 \\
\hline 0.251353 & 7.588235 & 0.200582 & 0.041992 & 19 & 410.875 \\
\hline 0.286489 & 10.47727 & 0.218002 & 0.050022 & 12 & 490.25 \\
\hline 0.309987 & 8.142857 & 0.229284 & 0.055664 & 9 & 550.5 \\
\hline
\end{tabular}

\section{Experimental Setup:}

The results are reported using 5-fold cross validation. K-nearest neighbor (kNN), Boosted Tree and SVM are used for classification.

\subsection{Experiment Using Combination Of Features:}

The experiments were performed with various combinations of features. The table 4 shows that Average Object Area, Euler Number is significant features. The

Combination of Average Object Area, Euler Number, Table 4: Accuracy (\%)

means and Area gives better result using SVM. The Performance of the two features i.e. entropy and Standard Deviation are not as good as Euler Number. The combination of Average Object Area
Euler Number, means and Area gives better result using KNN and Boosted Tree also as compared to another feature.

\begin{tabular}{|c|c|c|c|c|}
\hline $\begin{array}{l}\text { Sample } \\
\text { Size }\end{array}$ & Features & SVM & KNN & $\begin{array}{l}\text { Boosted } \\
\text { Tree } \\
\end{array}$ \\
\hline \multirow[t]{2}{*}{20} & $\begin{array}{c}\text { Average Object } \\
\text { Area+mean+Euler+Area }\end{array}$ & 87.9 & 82.9 & 86.7 \\
\hline & Euler & 86.3 & 64.1 & 86.3 \\
\hline \multirow[t]{2}{*}{15} & $\begin{array}{c}\text { Average Object } \\
\text { Area+mean+Euler+Area }\end{array}$ & 86.4 & 82.4 & 86.4 \\
\hline & Euler & 85.9 & 66.5 & 85.9 \\
\hline \multirow[t]{2}{*}{10} & $\begin{array}{c}\text { Average Object } \\
\text { Area+mean+Euler+Arean }\end{array}$ & 87.4 & 82.1 & 87.4 \\
\hline & Euler & 85.5 & 73.4 & 85.5 \\
\hline \multirow[t]{2}{*}{5} & $\begin{array}{c}\text { Average Object } \\
\text { Area+mean+Euler+Area }\end{array}$ & 84.4 & 81.1 & 84.4 \\
\hline & Euler & 83.5 & 73.6 & 83.5 \\
\hline
\end{tabular}

\subsection{Experiment with different sample size:}

The experiments were performed with different sample size. Accuracy increases with the sample size increase as shown in table 5 .

\subsection{Experiment with different signer:}

Table 6 shows Accuracy decreases with more signers.

\begin{tabular}{|c|c|c|c|c|}
\hline Users & Features & SVM & KNN & $\begin{array}{l}\text { Boosted } \\
\text { Tree }\end{array}$ \\
\hline \multirow[t]{2}{*}{10} & $\begin{array}{c}\text { Average Object } \\
\text { Area+mean+Euler No.+Area }\end{array}$ & 91 & 87.8 & 89.1 \\
\hline & Euler No. & 87.6 & 78.5 & 88 \\
\hline \multirow[t]{2}{*}{20} & $\begin{array}{l}\text { Average Object } \\
\text { Area+mean+Euler No.+Area }\end{array}$ & 90.2 & 88.9 & 89.2 \\
\hline & Euler No. & 88.2 & 80.7 & 87.9 \\
\hline \multirow[t]{2}{*}{30} & $\begin{array}{l}\text { Average Object } \\
\text { Area+mean+Euler No.+Area }\end{array}$ & 88.8 & 84.9 & 87.8 \\
\hline & Euler No. & 87.8 & 71.2 & 87.8 \\
\hline \multirow[t]{2}{*}{40} & $\begin{array}{l}\text { Average Object } \\
\text { Area+mean+Euler No.+Area }\end{array}$ & 89.0 & 83.4 & 87.7 \\
\hline & Euler No. & 87.8 & 67.0 & 87.9 \\
\hline
\end{tabular}

\subsection{Experiment with each user:}

The experiments were performed with each user using combination of

selected features (i.e. Average Object Area, Mean, Euler No., and Area) as mentioned in feature selection algorithm. Writer dependent model have more accuracy than writer independent model. For some user, we obtain $100 \%$ accuracy as table 7 shows.

\section{Conclusion}

In this paper, an intelligent algorithm for offline signature authentication has been presented. The algorithm is tested against CEDAR dataset using KNN, SVM and Boosted Tree. The comparison of the proposed algorithm with other research is generally considered a rather hard task due to various degrees of freedom regarding the type or number of signatures utilized during the training and testing phase. Proposed algorithm is quite efficient as it is less computationally. The numbers of signature pair used in WI model are less for each user as compared to other research .WD model gives better result than WI using proposed algorithm. 


\section{Acknowledgment}

The author would like to thank the CEDAR research group that built CEDAR signature dataset.

Table 7: Accuracy (\%) for each WI model

\begin{tabular}{|c|c|c|c|}
\hline User & Svm & Knn & Boosted Tree(Bag) \\
\hline 1 & 91.3 & 95.7 & 96 \\
\hline 2 & 89 & 91.3 & 93 \\
\hline 3 & 97.8 & 93.5 & 97.8 \\
\hline 4 & 93.5 & 91.3 & 93.5 \\
\hline 5 & 91.3 & 87 & 82.6 \\
\hline 6 & 97.8 & 95.7 & 95.7 \\
\hline 7 & 89.1 & 89.1 & 87 \\
\hline 8 & 91.3 & 91.3 & 91.3 \\
\hline 9 & 97.8 & 97.8 & 95.7 \\
\hline 10 & 100 & 100 & 100 \\
\hline 11 & 97.8 & 97.8 & 95.7 \\
\hline 12 & 97.8 & 93.5 & 97.8 \\
\hline 13 & 97.8 & 97.8 & 97.8 \\
\hline 14 & 100 & 100 & 97.8 \\
\hline 15 & 97.8 & 97.8 & 91.3 \\
\hline 16 & 97.8 & 93.5 & 97.8 \\
\hline 17 & 100 & 100 & 97.8 \\
\hline 18 & 100 & 100 & 97.8 \\
\hline 19 & 78.3 & 69.6 & 78.3 \\
\hline 20 & 93.5 & 84.8 & 93.5 \\
\hline 21 & 80.4 & 78.3 & 80.4 \\
\hline 22 & 67.4 & 69.6 & 63 \\
\hline 23 & 56.5 & 54.3 & 50 \\
\hline 24 & 93.5 & 93.5 & 93.5 \\
\hline 25 & 100 & 100 & 100 \\
\hline 26 & 89 & 89 & 89 \\
\hline 27 & 97.8 & 95.7 & 97 \\
\hline 28 & 100 & 97.8 & 89.1 \\
\hline 29 & 93.5 & 95.7 & 91.3 \\
\hline 30 & 97.8 & 97.8 & 97.8 \\
\hline 31 & 93.5 & 87 & 91.3 \\
\hline 32 & 100 & 100 & 100 \\
\hline 33 & 97.8 & 97.8 & 100 \\
\hline 34 & 97.8 & 97.7 & 97.8 \\
\hline 35 & 95.7 & 97.8 & 97.8 \\
\hline 36 & 91.3 & 78.3 & 87 \\
\hline 37 & 97.8 & 978 & 95.7 \\
\hline 38 & 93.5 & 95.7 & 97.8 \\
\hline 39 & 65.2 & 65.2 & 63 \\
\hline 40 & 87 & 84.8 & 84.8 \\
\hline 41 & 97.8 & 97.8 & 97.8 \\
\hline 42 & 71.7 & 73.9 & 84.8 \\
\hline 43 & 95.7 & 95.7 & 89.1 \\
\hline 44 & 89.1 & 82.6 & 89.1 \\
\hline 45 & 91.3 & 91.3 & 95.7 \\
\hline 46 & 100 & 100 & 97.8 \\
\hline 47 & 84.8 & 78.3 & 82.6 \\
\hline 48 & 69.6 & 65.2 & 67.4 \\
\hline 49 & 91.3 & 89.1 & 93.5 \\
\hline 50 & 97.8 & 93.5 & 95.7 \\
\hline 51 & 89.1 & 87 & 89.1 \\
\hline 52 & 84.8 & 80.4 & 82.6 \\
\hline 53 & 80.4 & 76.1 & 87 \\
\hline 54 & 97.8 & 95.7 & 97 \\
\hline 55 & 89 & 89 & 89 \\
\hline
\end{tabular}

\section{References}

[1] Kamlesh Kumari, V.K Shrivastava, " Factors Affecting the Accuracy of Automatic Signature Verification “,IEEE,2016

[2] Kamlesh Kumari, V.K Shrivastava, "A Review of Automatic Signature Verification", ICTCS,2016

[3] Kamlesh Kumari, Sanjeev Rana " Writer-Independent Off-Line Signature Verification", International Journal of Computer Engineering \& Technology (IJCET),9(4),2018,pp.85-89
[4] Boudamous, Feriel, et al. "An-open system for off-line handwritten signature identification and verification using histogram of templates and SVM." Advanced Technologies for Signal and Image Processing (ATSIP), 2017 International Conference on. IEEE, 2017.

[5] Longjam, Teressa, and Dakshina Ranjan Kisku. "A supervised manipuri offline signature verification system with global and local features." Embedded Computing and System Design (ISED), 2017

[6] Soleimani, Amir, Kazim Fouladi, and Babak N. Araabi. "Persian offline signature verification based on curvature and gradient histograms." Computer and Knowledge Engineering (ICCKE), 2016 6th International Conference on. IEEE, 2016.

[7] Kumar, Ashok, and Karamjit Bhatia. "A robust offline handwritten signature verification system using writer independent approach." Advances in Computing, Communication \& Automation (ICACCA2017), 3rd International Conference on. IEEE, 2017.

[8] Taşkiran, Murat, and Zehra Gülru Çam. "Offline signature identification via HOG features and artificial neural networks." Applied Machine Intelligence and Informatics (SAMI), 2017 IEEE 15th International Symposium on. IEEE, 2017.

[9] Harfiya, Latifa Nabila, Agus Wahyu Widodo, and Randy Cahya Wihandika. "Offline signature verification based on pyramid histogram of oriented gradient features." Informatics and Computational Sciences (ICICoS), 2017 1st International Conference on. IEEE, 2017.

[10] Okawa, Manabu. "Offline Signature Verification with VLAD Using Fused KAZE Features from Foreground and Background Signature Images." International Conference on Document Analysis and Recognition (ICDAR,. IEEE, 2017.

[11] Salama, Mostafa A., and Walid Hussein. "Invariant directional feature extraction and matching approach for robust off-line signature verification." Image, Vision and Computing (ICIVC), International Conference on. IEEE, 2016.

[12] Okawa, Manabu. "Offline signature verification based on bag-ofvisual words model using KAZE features and weighting schemes." Proceedings of the IEEE Conference on Computer Vision and Pattern Recognition Workshops. 2016.

[13] Pal, Srikanta, et al. "Performance of an off-line signature verification method based on texture features on a large indic-script signature dataset." 2016 12th IAPR Workshop on Document Analysis Systems (DAS). IEEE, 2016.

[14] Chauhan, Prachi, Subhash Chandra, and Sushila Maheshkar. "Static digital signature recognition and verification using neural networks." Information Processing (IICIP), 2016 1st India International Conference on. IEEE, 2016.

[15] Swapna, D., et al. "A survey on local patterns for signature verification." Communication and Signal Processing (ICCSP), 2016 International Conference on. IEEE, 2016.

[16] Chen, Siyuan, and Sargur Srihari. "Use of exterior contours and shape features in off-line signature verification." Document Analysis and Recognition, 2005. Proceedings. Eighth International Conference on. IEEE, 2005.

[17] www.cedar.buffalo.edu/NIJ/publications.html 